\title{
Multiple Beneficial Applications and Modes of Action of Growth Promoters in Rabbits Production
}

\author{
S. A. Abd EL-Latif \\ Department of Animal Production, Faculty of Agriculture, Minia University, Minia, Egypt \\ Corresponding author: profshaker71@yahoo.com
}

Recently, many countries tend to prevent the application of antibiotics for their side effects on both animal and human. Nowadays, growth promoters from herbal sources (phytogenic extracts) are used very commonly. Herbs such as Turmeric (Curcuma longa), ginger (Zingiber officinale), fenugreek (Trigonella foenum-graecum L.) and other herbs are phytogenic additives, possible alternatives, reported in poultry production which include a group of natural feed additives; derived from herbs, spices or other plants or their extracts in the form of essential oils. The optimum antioxidant activity of some herbs such as turmeric tends to stimulate protein synthesis by enzymatic system. Furthermore, herbs could control and limit the growth and colonization of numerous pathogenic and non-pathogenic species of bacteria in the rabbit's gut resulting in balanced gut microbial ecosystem that leads to better feed utilization reflected by live body weight and weight gain. Citrus have over 60 flavonoids and these compounds can be digested in the small intestine therefore, the level of these flavonoids can significantly increase in blood serum. Flavonoids have antioxidant, anti-inflammatory, antibacterial and immune-stimulating effects. The aim of this review was to evaluate the effect of growth promoters as feed supplementation in rabbit diets on some productive performances.

Keywords: growth promoters, productive performances, rabbits 